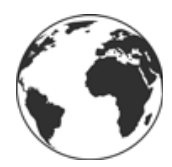

cambridge.org/enc
Thematic Section: Biodiversity Revisited

\section{The $A B C$ of planetary insecurity: a crisis in need of system acupuncture}

Banny Banerjee (1)

Stanford ChangeLabs, Stanford, CA, USA

\section{Comment}

Cite this article: Banerjee $B$ (2021) The $A B C$ of planetary insecurity: a crisis in need of system acupuncture. Environmental Conservation 48: 71-74. doi: 10.1017/S0376892921000072

Received: 25 May 2020

Revised: 26 January 2021

Accepted: 27 January 2021

First published online: 8 March 2021

\section{Author for correspondence:}

Banny Banerjee,

Email: banny@stanfordalumni.org (c) The Author(s), 2021. Published by Cambridge University Press on behalf of Foundation for Environmental Conservation.
At least three of the nine planetary boundaries for a safe operating space for humanity have been crossed (biodiversity loss, climate change and nitrogen balance), alongside insufficient understanding of the status, interrelations or collective implications of the others (Rockström et al. 2009). Despite decades of scientific caution about limits to growth and human contribution to climate change, there continues to be a profound gap between scientific knowledge and requisite action. Inadequacy of concerted and transformative action represents a risk to intergenerational wellbeing (Arrow et al. 2012). With the objective of addressing critical gaps, failure modes and deficiencies in current responses to complex planetary challenges, this paper (1) reframes the challenge, (2) identifies structural gaps in current approaches and (3) proposes System Acupuncture as an approach for bringing about system transformation at scale.

\section{Reframing the challenge: the $\mathrm{ABC}$ of Planetary Insecurity}

The way a challenge is framed dictates the directionality and structure of the response. Progress framed in terms of gross domestic product (GDP) overemphasizes economic indicators while deprioritizing long-term planetary resilience.

This paper introduces the frame 'Planetary Insecurity' as the cumulative threats and loss of resilience in critical social-environmental systems (Reid et al. 2018) and proposes a single overarching systems challenge: the 'ABC of Planetary Insecurity' (in short, the 'ABC Crisis'): Anthropocene Unchecked $\times$ Biodiversity Collapse $\times$ Climate Crisis as a single interrelated triad, representing a unified systemic threat to life on Earth and human civilization. The response to the $\mathrm{ABC}$ Crisis determines the trajectory of Planetary Insecurity.

The 'ABC Crisis' is framed as the product of three challenges:

(1) 'Anthropocene Unchecked' refers to the systemic way in which human activity extracts planetary resources faster than their recharge rates and pushes past planetary boundaries, tipping the biosphere towards increased instability.

(2) 'Biodiversity Collapse' refers to unprecedented rates of biodiversity loss, both an indication and a cause of rapid destruction of the intricate web of dependencies that maintains ecological stability, a core condition for life and civilization.

(3) 'Climate Crisis' refers to shifts in climatic patterns causing rapidly escalating threats to safe operating conditions for life-forms including humans.

Planetary Insecurity is expressed as a product of these three multi-dimensional factors, conceptually similar to a vector cross product in linear algebra where the resultant is another directional vector. At a conceptual level, it can be expressed as:

$[$ Planetary Insecurity $]=[$ Anthropocene Unchecked $] \times[$ Biodiversity Collapse $] \times[$ Climate Crisis $]$

The square brackets remind us that the terms are multi-dimensional.

Humankind's entrenchment in an 'anthropocentric' rather than 'planet-centric' frame threatens intergenerational wellbeing. Short-term human concerns dominate decisions and actions, rarely considering longer-term system impacts. Economic concerns dominate social ones, and social concerns overpower environmental and biodiversity issues. Take food security as an example: despite innovative regenerative agricultural techniques with higher yields on already converted land, current market forces and government policies drive rapid deforestation (Pearson 2007). The choice of achieving food security at the cost of biodiversity is a manifestation of the Anthropocene marching on unchecked.

Losing a single species is irreversible and can undermine a delicate web of ecological interdependencies - it is akin to throwing out components of a clock whilst still depending on the clock to tell the time. The scale of the current biodiversity collapse indicates that we are in the 
midst of the sixth mass extinction (Ceballos et al. 2015). Civilization depends on the stability of the biosphere, which is undergoing 'Biological Annihilation' (Ceballos et al. 2017).

Meanwhile, despite the target of limiting the rise of mean global temperatures to $2^{\circ} \mathrm{C}$ above pre-industrial levels, most climate models project a rise of $3^{\circ} \mathrm{C}$ by 2100 (uncertainty range $2.0-5.4^{\circ} \mathrm{C}$ ). Apart from dire projected consequences in the next few decades, the variability experienced at localized levels is already causing violent and devastating impact. Given an inadequate global response, the temporal and the critical aspects of the challenge need emphasis - hence the term 'Climate Crisis', underscoring the exigency, rather than mean global temperatures and $\mathrm{CO}_{2}$ levels.

Factors perpetuating the ABC Crisis are systemic and extremely complex. Therefore, a systems lens is needed to address Planetary Insecurity.

\section{Identifying the gap: mechanistic approaches for a systems crisis}

The 'ABC Crisis' extends the conceptualization from a 'super-wicked problem' (Levin et al. 2012) to a 'hyper-wicked challenge' - a single, non-linear, overarching challenge with multiple interrelated superwicked problems adding up to critical instabilities at the planetary level. It cannot be tackled with traditional problem-solving techniques (Rockström et al. 2010) or epistemological norms. Systems challenges need systems approaches, scaled challenges need scaled approaches, urgent challenges require immediate action and non-linear challenges need non-linear transformations. Approaching a systems challenge without a systems perspective is like playing soccer with a javelin.

Key structural shortcomings of traditional approaches include the following:

O Process mismatch - deterministic and mechanistic thinking being used for systemic challenges that require systems approaches;

O Hyperbolic discounting - valuing the present disproportionately over the future;

Framing gap - success framed through reductive and piecemeal metrics without aiming for systemic outcomes;

$O$ Enquiry and understanding gap - disciplines working in silos with little enquiry into the interrelations or system dynamics;

Innovation gap - selection from existing solutions, however ineffectual, rather than designing new disproportionately effective ones;

O Leadership gap - promoting incrementalism rather than driving systemic outcomes;

O Coordination gap - lack of coordination across agencies, disciplines (Redman et al. 2004), stakeholders and institutions;

O Resource direction gap - resources directed at mitigating Planetary Insecurity are insufficient given the magnitude of the challenge and are dwarfed by those driving the problem; and

O Action gap - actions being too little, too late, or ineffectual, and held hostage by decision gridlocks.

These shortcomings in conventional approaches impede systemic outcomes and allow crises to escalate whilst draining scarce resources. A paradigmatic shift in approach is needed to deliver planetary-scale system transformation in unprecedented timeframes. This paper proposes 'System Acupuncture' as a methodological framework and a structure of practice for addressing complex systems challenges, taking into account diverse motivations and competing objectives.

\section{Innovating systems transformation: System Acupuncture}

System Acupuncture borrows a metaphor from traditional Chinese medical practice. Instead of brute force techniques, the notion is to identify 'acupuncture points' in the system that yield disproportionate leverage in transforming system trajectories. It is used to design a portfolio of innovative, transformative, actionable and synergistic interventions to bring about system transformation in accelerated timeframes. It steers a complex system towards desirable, resilient, sustainable and self-healing futures, seeking next-generation paradigms and models, while avoiding unintended negative consequences (Fig. 1).

\section{System Acupuncture as a multifaceted approach}

The goal of System Acupuncture is to create a 'portfolio' of actionable 'intervention pathways' that alter the behaviour of the system so that its natural outcomes are the desired ones. An example of a pathway would be a new market instrument that places economic value on continual ecological functioning of unconverted forests, thus driving investments away from land conversion for agriculture and towards regenerative farming.

A portfolio with multiple powerful interventions needs to consider and navigate their delicate interplay or temporal staging. It should focus on maximizing their mutual reinforcement while minimizing negative influences on each other or on system outcomes. Since system interactions of interventions are difficult to predict in their entirety, system innovation work relies on incorporating an iterative and navigational innovation management approach.

System Acupuncture calls for some key ingredients to be combined in strategic and multiplicative ways:

(1) Systems Leadership: a leadership stance Systems Leadership reframes leadership from a role to a modality, allowing for distributed ways of catalysing change. Apart from a departure from hierarchical models of leadership, a key distinction lies in creating a distributed leadership function and maintaining a relational 'complex systems' mind-set. Systems Leaders create 'transformation capacity' by building ecosystems and governance systems to enable 'end-to-end' system-level transformation. They introduce a systems perspective to the way in which change is framed, envisioned, initiated, designed, directed, amplified, implemented, navigated and ultimately managed.

(2) Systems Innovation methodology: a Process for scaled system transformation

The role of innovation is to find creative ways of disproportionately outperforming normative means and generating new possibilities. Systems Innovation methodology guides transdisciplinary and trans-agency (Banerjee et al. 2014) groups of stakeholders to co-create a 'Systems Theory of Change' and actionable intervention pathways with the potential to transform the system at scale. System Innovation combines all available resources with unprecedented leverage - scientific insights, social insights, non-linear technologies, market forces, business models, organizational capacity, networks, behaviour change, governance systems, policy, data, etc. It generates strong value propositions for mainstream players to be a part of the solution rather than the problem.

(3) System Change Platforms: enablers of non-linear organization Platforms, through their non-linear structure, can rapidly integrate broad engagement, self-propelling strategies, 


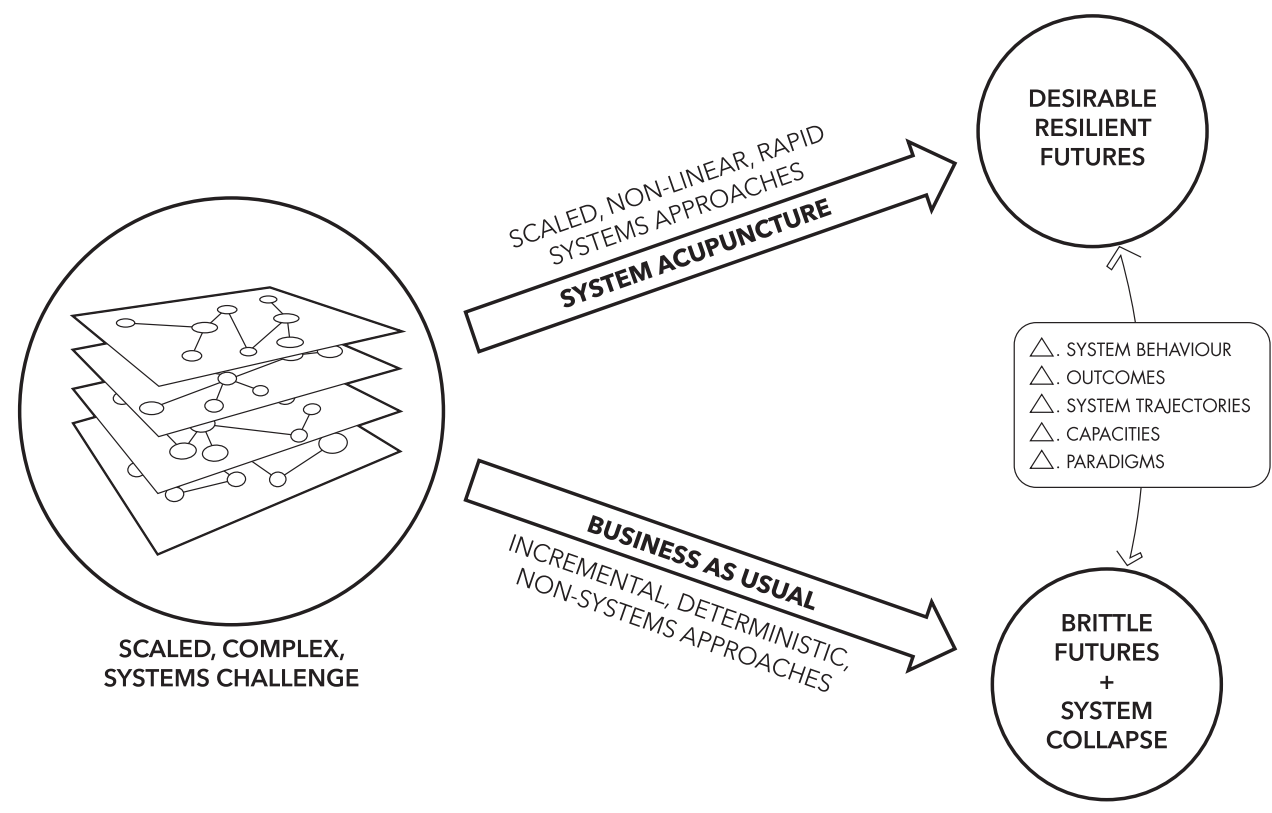

Fig. 1. The Wicked Fork: the choice facing humanity between its current trajectory leading to increased Planetary Insecurity and an alternative one leading to inter-generational well-being. System Acupuncture is proposed as a philosophy and an approach to drive rapid system transformation given conflicting needs, realworld constraints and resource limitations. non-linear technology, monitoring and resource redirection with continually lowered transaction costs. System Change Platforms bridge chasms between seemingly contradictory motivations and contextual realities. They are designed to maintain the cogency of strategic direction in cross-sector stakeholder networks. For instance, national- and regionallevel networks of Sustainable Development Goal (SDG) platforms could drive much-needed cross-ministry and cross-industry action to uncover systemic change opportunities to meet the SDGs.

(4) System Change Resources: directed and leveraged flow of resources

In a systems approach, resources are defined in broader ways to encompass institutional power, hybrid funding, innovative finance, political will, strategic policy, leadership capacity, innovation capacity, expertise, technology, information, relationships, human behaviour, waste streams, stakeholder engagement, incentives, cultural norms, narratives and networks. System Acupuncture alters patterns of resource flows to restructure and amplify the intended system behaviour. For example, with overfishing causing ecological devastation, the combination of a 'forward market', dynamic zoning, dynamic pricing based on catch location and fishers being co-invested in a new financial instrument such as a 'healthy fish stock' would create new markets and incentives to align fishing behaviour while regenerating marine biodiversity.

(5) System Change Action: a modality of action

Without action, the best system strategy is merely a thought experiment. Scaled challenges require an action repertoire and agency that actively disrupts existing system dynamics and replaces them with alternatives. System Change Action engages multi-stakeholder networks to commit, navigate and amplify system-level impact. The action is shaped to gainfully pull larger and larger parts of the system to align with new norms and models.

The System Acupuncture components listed above imply new capabilities, mind-sets, decision frames, organizational structures and workflows. Instead of allowing a lack of capacity to preclude action, the application of System Acupuncture is a framework for rapidly acquiring system transformation capacity on the path to making impact. Participating in the co-creative process allows stakeholders to incorporate systems principles into their culture of practice and build the capacity to manage and navigate systemlevel transformation.

Mitigating the $\mathrm{ABC}$ Crisis demands collective efforts of stakeholders with diverse perspectives and motivations. System Acupuncture provides the theoretical underpinning and the practical tools to enable stakeholders across the system to drive scaled impact in the face of immense complexity.

\section{A global emphasis on systems transformation}

The $\mathrm{ABC}$ Crisis is a juggernaut with a diminishing window of opportunity for effective intervention. Delayed action, incrementalism and relying on traditional problem-solving approaches constitutes being complicit with system failure. This unprecedented class of challenge calls for a sense of urgency, humility regarding the limited efficacy of current approaches and aggressiveness in exploring and conceptualizing actionable pathways towards system-level transformation.

Acknowledgements. The author thanks Annette Zou, Stanford ChangeLabs, and Carina Wyborn, WWF.

Financial support. This research received no specific grant from any funding agency, commercial or not-for-profit sector.

Conflict of interest. None.

Ethical standards. None.

\section{References}

Arrow KJ, Dasgupta P, Goulder LH, Mumford KJ, Oleson K (2012) Sustainability and the measurement of wealth, Environment and Development Economics 17: 317-353.

Banerjee B (2014) Large scale integrated innovation. In: C Bason (ed.), Design for Policy (pp. 71-87). New York, NY, USA: Routledge. 
Ceballos G, Ehrlich PR, Barnosky AD, Garcia A, Pringle RM, Palmer TM (2015) Accelerated modern human-induced species losses: entering the sixth mass extinction. Science Advances 1: 1-5.

Ceballos G, Ehrlich PR, Dirzo R (2017) Biological annihilation via the ongoing sixth mass extinction signaled by vertebrate population losses and declines. Proceedings of the National Academy of Sciences of the United States of America 114: E6089-E6096.

Levin K, Cashore B, Bernstein S, Auld G (2012) Overcoming the tragedy of super wicked problems: constraining our future selves to ameliorate global climate change. Policy Sciences 45: 123-152.

Pearson CJ (2007) Regenerative, semiclosed systems: a priority for twenty-firstcentury agriculture. Bioscience 57: 409-418.
Redman CL, Grove JM, Kuby LH (2004) Integrating social science into the Long-Term Ecological Research (LTER) network: social dimensions of ecological change and ecological dimensions of social change. Ecosystems 7: 161-171.

Reid WV, Chen D, Goldfarb L, Hackmann H, Lee YT, Mokhele K et al. (2018) Social-ecological systems insights for navigating the dynamics of the Anthropocene. Annual Review of Environment and Resources 43: 267-289.

Rockström J, Schellnhuber HJ, Whyte A (2010) Earth system science for global sustainability: grand challenges. Science 330: 916-917.

Rockström J, Steffen W, Noone K, Persson A, Chapin FS, Lambin E et al. (2009) A safe operating space for humanity. Nature 461: 472-475. 